\title{
INTEGRAL EQUATION ANALYSIS OF DRIFT WAVE EIGENMODES IN A SHEARED SLAB GEDHETRY
}

MASTER

BY

W. M. TANG, G, REWOLDT. AND E, A, FRIEMAN

\section{PLASMA PHYSICS LABORATORY}

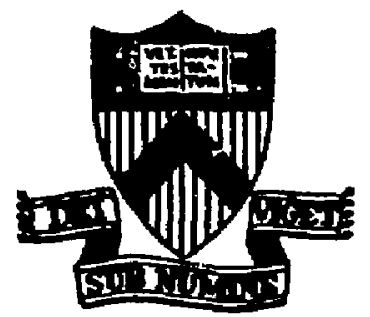

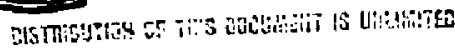

\section{PRINCETON UNIVERSITY PRINCETON, NEW JERSEY}

This work was supported by the $\dot{U}$. $s$. Department of Energy Contract Ho. EY-76-0-02-3073. Aeproduction, translation, publication, use and disposal, in whole or in part, by or for the Dafted States fovernient is pernitted. 
Integral Equation Analysis of Dritt wave Einenmodes in a Sheared slab Geometry

W. M. Tang, G. Rewoldt, and E. A. Frieman

PJasma Physics Laboratory, Princeton University, Princeton, New Jersey 08544

The derivation of the appropriate form for the integral eigenmode equation governing both electron and ion drift waves of arbitrary radial wavelengths in a sheared slab is presented. The solutions to this equation provide useful information regarding the absolute stability of universal modes and iontemperature-gradient driven modes for arbitrary wavelengthe; and particularly for short wavelengths.

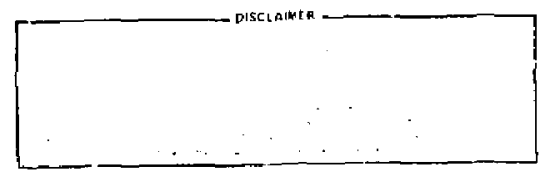




\section{INTRODUCTION}

The investigation of the stability of drift waves in a sheared slab geometry has been actively pursued in numerous recent publications. ${ }^{1-3}$ These calculations have dealt with long radial wavelength eigenmodes governed by a differential equation of the form

$$
\left[p_{i}^{2} \partial^{2} / \partial x^{2}+Q(x, \omega)\right] \bar{\beta}(x)=0
$$

with $\rho_{i}$ being the ion gyroradius and $Q(x, t)$ boing the racial potential whose specific form is determined by the particular instability considered. llowever, at shorter radial wavelengths, where the assumption $\left|\rho_{i}^{2} \partial^{2} / \lambda x^{2}\right| \ll 1$ breaks down, it becomes necessary to deal with the integral equation generalization of Eq. (1) The present paper is concerned with the derivation of the appropriate form of this integral $c$-jerinode equation and with obtaining solutions for both electron and ion drift waves.

Results from the differential eigenmode equation studies have indicated that as the azimuthal wave number $\left\langle k_{y}\right\rangle$ is increased to $k_{y} p_{i}-1$, the radial localization of the mode becomes of the order of the ion gyroradius. For electron drift waves, ${ }^{l}$ this is a consequence of the fact that as $k_{Y} p_{i}$ is increased, there is a downward shift of the eigenfrequency caused by finite gyroradius effects; i. $e_{1} \omega=a_{\star} \mathrm{e}_{0}\left(\mathrm{~b}_{y}\right)$ exp $\left(-\mathrm{b}_{Y}\right)$ with $h_{y}=k_{y}^{2} p_{i}^{2} / 2$ and $I_{0}$ being the familfiar Bessel function. since the radial position, where ion-fiandau dpmping becomes dominant, is proportional to $\omega$ li.e., $\omega \cong k_{1} v_{i}$ wifh $v_{i}$ being the ion thermal 
velocity and $k_{11} \propto x$ for a sheared $\left.s l a b\right)$, the corresponding radial localization of the eigermode is also rhifted to smaller values. Hence, it is of interest to apply the proper integral equation analysis to examine the stability properties of these waves at shorter wavelengths.

In the case of ion drift waves, ${ }^{2,3}$ the radial locallzation of the eigenmodes is again determined by the ion-iandau resonarce region. Differential equation eigenmode studies of ion-temperaturegradient-driven instabilities of this branch indicate that the largest qrowth rates are found for waves with $k_{y}{ }_{i}$ approaching one with corresponding eige.imodes localized to a radial extent of a few ion gyroradji. 2,3 since the dominant part of the unstable spectrum here falls in the regime of wavelengths where the diffurential eigenmode equation is breaking down, it becomes important to generalize the calculation to an integral equation analysis appropriate at arbitrary wavelengths.

Motivated by the preceding considerations, we present a systematic derivation of the integral eigenmode equation of interest in 5ec. II. In this section the procodures employed to solve the resultant equation are also described. Results from the computer code implementing the integral equation formalism ("integral formulation code") are presented in sec. III for collisionless electron drift waves ("universal" modes), and in Sec. IV for the ion-temperature-gradient-driven ion drift instabilities. Finally, we conclude with a brief summary and general comnents in sec. $v$. 


\section{Derivation of Integral Eigenmode Equation}

The torm of the integral equation governing collisioniess electrostatic drift waves for arbitrary ${ }^{\prime} x^{\prime}{ }^{\prime} i$ in a sheared slab geometry is derived in this section. Assuming that there is no equilibrium electric field, the governing equation for the perturbed distribution function $t$ (given, for example, in

Ref. 4) reduces to:

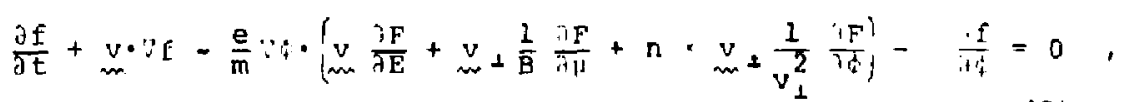

where $F=F_{m}+F^{(1)}$ is the equilibrium distribution function, : is the gyrophase angle, $E$ is the kinctic energy per unit mass, $\mu$ is the magnetic moment per unit mass, $\phi$ is the perturhed electrostatic potential, an $\Omega \equiv \mathrm{eB} / \mathrm{mc}$. Species subscripts are suppressed here. We deal with a sheared magnetic field given by ${ }_{o r}=B_{7}$ e + $B_{y}(x) \underbrace{}_{m y}$, and consider the ordering

$$
\frac{k_{1}}{k_{1}}=O(E), \frac{B_{Y}}{B_{2}}=O(E)
$$

with $\varepsilon$ being the fundamental smallness parameter and $B_{y}$ taken to be proportional to $x$. Hence, $B=B_{z}$, and $n \equiv B / B=n_{m}(0)+n_{m}(1)$, with $n_{m}^{(B)}=e_{m}$ ard $_{m}{ }^{(1)}=\left(B_{y^{\prime}} / B\right)$ e $y^{\prime}$ where the superscripts 
refer to the order in $\varepsilon$. Thus $n^{(1)} / n^{(0)}=O(\varepsilon)$. $F_{m}$ is a Maxwellian and, from Ref. 4,

$$
F^{(1)}=\frac{v_{\perp}}{\Omega} \sin \phi \frac{\partial F_{m}}{\partial(\varepsilon x)} .
$$

To lowest order in $[, E q .(2)$ becomes

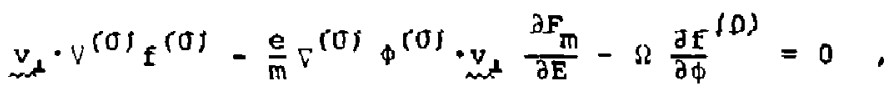

with the notation $\nabla_{1}^{(0)}=\partial / \partial x_{m}, \nabla_{1}^{(1)}=\partial / \partial(c x)$, and $\nabla_{z}=\nabla_{z}^{(1)}=\partial / \partial z$. Defining $h^{(0)}$ by

$$
f^{(0)}=\frac{e}{m} \phi(0) \frac{\partial F_{m}}{\partial E}+h^{(0)}
$$

Eq. (5) becomes

$$
v_{n+} \cdot \nabla^{(0)} h^{(0)}-\Omega \frac{\partial}{\partial \phi} h^{(0)}=0 .
$$

Without loss of generality, we can express $h^{(0)}$ in the form:

$$
h^{(0)}=(2 \pi)^{-1 / 2} \int_{-\infty}^{\infty} d k_{x} \bar{h}\left(E, \mu_{r} \phi, k_{x}\right) \exp i\left(k_{x} x+k_{z} z-\omega t\right) .
$$


Hence Eq. (7) becomes

$\left(i k_{x} v_{\perp} \cos \phi+i k_{y} v_{\perp} \sin \phi-2 d / \partial \phi\right) \bar{h} \exp i\left(k_{x} x+k_{y} y\right)=0$.

Letting $\bar{h}=\hat{h}^{(0)}\left(E, i, k_{x}\right) g(t)$ gives

$$
\frac{i k_{x} v_{\perp}}{\Omega} \cos d+\frac{i k y_{\perp}}{\Omega} \sin \psi=\frac{1}{g} \frac{u}{i \phi} g \text {. }
$$

so that

$$
g(\phi)=\exp \left[i \frac{v_{1}}{\Omega}\left(k_{x} \sin \phi-k_{y} \cos \phi\right)\right]
$$

From Eq. (8), we then get

$$
n^{(0)}=(2 \pi)^{-1 / 2} \int_{-\infty}^{\omega x} d k_{x} \hat{h}^{(0)}\left(E, \mu, k_{x}\right) \exp i\left(k_{x} x+k_{y} y+k_{z} z-\omega t+L\right) \text {, }
$$

with $L=\left(v_{1} / \Omega\right)\left(k_{x} \sin \phi-k_{y} \cos \phi\right)$. Now we need to determine $\hat{h}^{(0)}$ from the first order form of Eq. (2), which becomus:

$$
\begin{aligned}
& \frac{\partial f}{\partial t}^{(0)}+v_{11} n^{(0)} \cdot \nabla^{(1)} f^{(0)}+v_{11} n^{(1)} \cdot v^{(0)} f^{(0)}+v_{m} \cdot \nabla^{(1)} f^{(0)}+v_{1} \cdot v^{(0)} f^{(1)}
\end{aligned}
$$

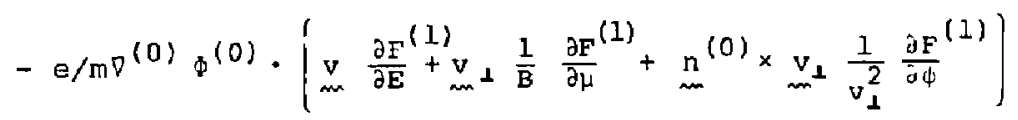

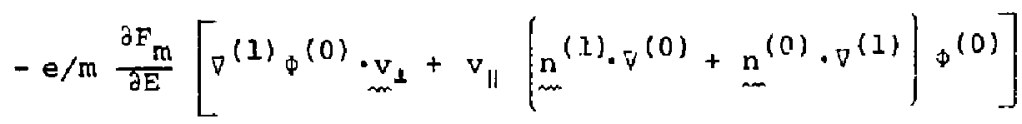

$$
\begin{aligned}
& -\Omega \frac{\partial f}{\partial \phi}^{(1)}=0 .
\end{aligned}
$$


In terms of $h^{(0)}$ this becomes

$$
\begin{aligned}
& -i \omega h(0)+\frac{e}{m} \frac{\partial \phi}{\partial t}^{(0)} \frac{\partial F_{m}}{\partial E}+v_{11} n^{(0)} \cdot \nabla^{(1)} h^{(0)}+v_{11}{ }^{(1)} \cdot \nabla^{(0)} h^{(0)} \\
& +v_{x} \cdot \nabla^{(1)_{h}(0)}+\frac{e}{m} \phi(0) v_{\mu^{2}} \cdot \nabla^{(1)} \frac{\partial F_{m}}{\partial \bar{E}}+v_{d} \cdot \nabla^{(0)} f^{(1)}-\frac{e}{m} \nabla^{(0)} \phi^{(0)} \\
& \cdot\left[v \frac{\partial F}{\partial E}^{(1)}+v_{\alpha} \frac{1}{B} \frac{\partial F}{\partial \mu}^{(1)}+\Omega^{(0)} \times y_{\alpha} \frac{1}{v_{l}^{2}} \frac{\partial F}{\partial \phi}^{(1)}\right]-\Omega \frac{\partial}{\partial \phi} f^{(1)}=0 \text {. }
\end{aligned}
$$

Again without loss of generality we can express $\phi(0)$ in the form:

$$
\phi^{(0)}=(2 \pi)^{-1 / 2} \int_{-\infty}^{a} d k_{x} \hat{\phi}^{(0)}\left(k_{x}\right) \exp j\left(k_{x} x+k_{y} y+k_{z} z-\omega t\right) .
$$

Using Eg. (12) for $h^{(0)}$ and expressing $f^{(1)}$ in the form:

$$
f^{(1)}=(2 \pi)^{-1 / 2} \int_{-\infty}^{\infty} d k_{x} f^{(1)}\left(E, \mu, \phi, k_{x} l \text { exp } i\left(k_{x} x+k_{y} y+k_{z} z-\omega t+L\right)\right. \text {, }
$$

Eq. (14) reduces to the following forn after averaging over the gyrophase angle $\phi$ : 
$-8-$

$$
\begin{aligned}
& -i \omega \hat{h}^{(0)}+i \omega \frac{\hat{e \phi}}{T} \hat{\sigma}^{(0)} F_{m} J_{0}\left(k_{\perp} v_{\perp} / \Omega\right)+i k_{z} v_{\| l} \hat{h}^{(0)} \\
& +i k_{y} v_{\|} \frac{B_{y}}{B} \hat{h}^{(0)}+\frac{e}{m} \hat{\phi}^{(0)}\left\langlee ^ { - i L } \left\{ v_{\lambda} \cos \phi \frac{\partial^{2} F_{m}}{\partial E \partial\left(E_{x}\right)}\right.\right.
\end{aligned}
$$

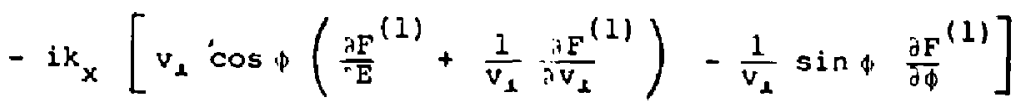

$$
\begin{aligned}
& \left.\left.+i k_{y}\left[v_{\perp} \sin \phi\left(\frac{\partial F^{(1)}}{\partial E}+\frac{1}{v_{\perp}} \frac{F^{(1)}}{\partial v_{\perp}}+\frac{1}{v_{\perp}} \cos \phi{\frac{1 F}{v_{\perp}}}^{(1)}\right)\right]\right\}\right\rangle=0 \text {. } \\
& \text { (17) } \\
& \langle\cdot .\rangle \equiv \frac{1}{\partial \pi} \int_{b}^{2 \pi} d . . .
\end{aligned}
$$

Substituting for $F^{(1)}$ from Eg. (4), the last term in EGg. (17) becomes 
$-9-$

$$
\begin{aligned}
& \frac{e}{m} \hat{\phi}(0)\left(e ^ { - i L } \left[v_{2} \cos \phi \frac{\partial^{2} F_{m}}{\partial E \partial(E x)}-i k_{x} \frac{v_{\perp}^{2}}{\hat{s}} \cos \phi \sin \phi \frac{\partial^{2} F_{m}}{\lambda E \partial(c x)}\right.\right. \\
& \left.\left.-i k_{y}\left(\frac{v_{\perp}^{2}}{\Omega} \sin ^{2} \phi \frac{\partial^{2} \mathbf{F}_{m}}{\partial E^{2}(\varepsilon x)}+\frac{1}{\Omega} \frac{\partial F_{m}}{\partial(\varepsilon x)}\right)\right]\right) \\
& =-\frac{e}{m} \hat{\phi}^{(0)} \frac{i k y}{\Omega} \frac{\partial F_{m}}{\partial(E x)}\left(e^{-i L}\right)+\frac{e}{m} \hat{\phi}^{(0)} \frac{\partial^{2} F_{m}}{\partial E \lambda(\varepsilon x)}\left\langle e^{-i L}\right. \\
& \left.\times\left[v_{\perp} \cos \phi-i\left(k_{x} \cos \phi+k_{y} \sin \phi\right) \frac{v_{\perp}^{2}}{\Omega} \sin \phi\right]\right) \\
& =-\frac{e}{m} \hat{\phi}(0) \frac{i k y}{\Omega} \frac{\partial F_{m}}{\partial(E x)} J_{0}\left(\frac{k_{+} v_{2}}{\Omega}\right)+\frac{e}{m} \hat{\phi}^{(0)} \frac{\eta^{2} F_{m}}{i E j(\varepsilon x)} \\
& x v_{\perp}\left(\frac{\partial}{\partial \phi}\left\{\sin \phi \exp \left[i \frac{v_{1}}{\Omega}\left(k_{y} \cos \phi-k_{x} \sin i\right)\right]\right\}\right) \\
& =-\frac{\mathrm{e}}{\mathrm{m}} \hat{\phi}(0) \frac{i k_{Y}}{\Omega} \frac{\partial \mathrm{F}_{\mathrm{m}}}{\mathrm{d}(E \mathrm{Ex})} J_{0}\left(\frac{k_{\perp} v_{1}}{\Omega}\right) .
\end{aligned}
$$

So, Eq. (17) reduces to

$$
\hat{h}^{(0)}=\frac{e \hat{\phi}}{T}^{(0)} F_{m} J_{0}\left(\frac{k_{\perp} v_{1}}{\Omega}\right)\left(\frac{\omega-\omega^{T}}{\omega-k_{11} v_{11}}\right),
$$


with $k_{\|} \equiv k_{z}+k_{y} B_{y} / B_{*} \omega_{*}^{T} \equiv \omega_{*}(1+n(m \varepsilon / T-3 / 2)]$,

$n \equiv(d \ln T / d x) /(d \ln n / d x), w_{*} \equiv-k_{y}\left(c T / e B L_{n}\right)$ and $L_{n} \equiv$

$-(d \ln n / d x)^{-1}$. With $\hat{h}^{(0)}$ thus determined, Eq. (12)

yields:

$$
\begin{aligned}
h(0) & =\frac{e}{T} F_{m}\left(\frac{\omega-\omega^{T}}{\omega-k_{11} v_{11}}\right) \exp i\left(k_{y} y+k_{z} z-\omega t\right)(2 \pi)^{-1 / 2} \\
& \times \int_{-\infty}^{m} d k_{x}{ }^{(0)}\left(k_{x}\right) J_{0}\left(\frac{k_{\perp} v_{\perp}}{\zeta}\right) \exp i\left(k_{x}+L\right) .
\end{aligned}
$$

Finally, the gyrophase-averaged perturbed distribution function can be expressed as

$$
\begin{aligned}
\left\langle f^{(0)}\right\rangle= & =\frac{e^{\phi}}{T}{ }^{(0)} F_{m}+\frac{e}{T} F_{m}\left(\frac{w-w^{2}}{w-k_{11} v_{11}}\right)(2 \pi)^{-1 / 2} \int_{-\infty}^{a} d k_{x} \\
& \times \phi^{(0)}\left(k_{x}\right) J_{0}^{2}\left(\frac{k_{x} v_{\perp}}{\Omega}\right) \exp i\left(k_{x} x+k_{y} y+k_{z} z-w t\right) .
\end{aligned}
$$

If we write $\mathrm{B}_{\mathrm{y}} / \mathrm{B}=\mathrm{x} / \mathrm{L}_{\mathrm{s}}$ and take $\mathrm{k}_{\mathrm{z}}=0$ (which is equivalent to a change in origion of $x\rangle$, then $k_{11}=k_{y} x / L_{s} \equiv k_{i j} x$. 
We will use the notation $\phi(0)=\dot{\phi}(x) \exp i\left(k_{y} y-\omega t\right)$ for

the perturbed electrostatic potential and $n_{j}^{(0)}=\tilde{n}_{j}(x) \exp i\left(k_{y} y-w t\right)$ for the perturbed density, where $j=i$ for ions with charge $e$ and $j=e$ for electrons with charge - e. Carrying out the velocity space integrations of Eq. (20) gives

$$
\begin{aligned}
& \dot{n}_{i}=-\frac{e}{T} n\left[\tilde{\phi}(x)+\left(\frac{1}{v_{i} \bar{k}_{j}|x|} z\left(\xi_{j}\right)(2 \pi)^{-1 / 2} \int_{-\infty}^{\infty} d k_{x} \exp \left(i k_{x} x\right)\right.\right. \\
& \left.\left.x\left|\left(w-w_{* i}\left(1-\frac{3}{2} n_{i}\right)\right) r_{0}-w_{i} n_{i}\right| r_{0}+b\left(r_{1}-\Gamma_{0}\right)\right]\right\} \\
& \left.-\frac{\omega_{i} r_{i}}{v_{i} k_{i}|x|}\left[\xi_{i}+r_{i}^{2} z\left(\xi_{i}\right)\right](2 \pi)^{-1 / 2} \int_{-\infty}^{m} d k_{x} \exp \left(i k_{x} x\right) \Gamma_{u}\right) \\
& \left.x(2 \pi)^{-1 / 2} \int_{-\infty}^{m} d x \exp \left(-i k_{x} x\right) \tilde{\phi}(x)\right] .
\end{aligned}
$$

and

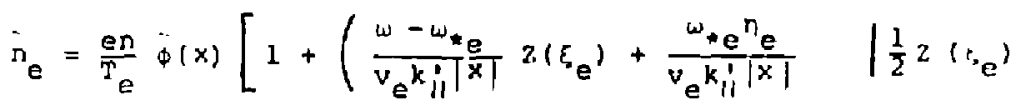

$$
\begin{aligned}
& \left.\left.\left.-\xi_{e}-\xi_{e}^{2} z\left(\xi_{e}\right)\right]\right)\right] \text {. }
\end{aligned}
$$

Here $v_{j} \equiv\left(2 \mathrm{~T}_{j} / \mathrm{m}_{j}\right)^{1 / 2}, \xi_{j} \equiv \omega /\left(v_{j} k_{i}|\mathrm{x}|\right), r_{0,1}: I_{, L}(\mathrm{~b}) \exp (-\mathrm{b})$, $I_{o}$ and $I_{1}$ are modified Bessel functions of the first kind, $b \equiv\left(k_{x}^{2}+k_{y}^{2}\right) \rho_{i}^{2} / 2 \equiv b_{x}+b_{y}, \rho_{i} \equiv v_{i} / \Omega_{i}$, and $z$ is the usual plasma 
dispersion function. In computing $\tilde{n}_{e} \cdot k_{1} p_{e}=k_{1} v_{e} / t_{e}$ has been assumed to be negligible. Although Eqs. (21) and (27) are valid only in the collisionless limit, the addition of electron collisions by means of a number-conserving Krook model is straightforward.

The equation to be solved for the normal modes is just the quasi-neutrality condition,

$$
0=n_{e}-n_{i}
$$

This equation can be solved by means of a Ritz mothod. First $(y)$ is decomposed into appropriate basis functions:

$$
\tilde{\phi}(x)=\sum_{n=n}^{\infty} \Phi_{n} h_{n}(x),
$$

where

$h_{n}(x)=H_{n}\left(0^{1 / 2} k_{y} s x\right)$ exp $\left(-a k_{Y}^{2} s^{2} x^{2} / 2\right) M_{n}^{-1 / 2}$

$\hat{s} \equiv\left(q^{\prime} r / q\right)$ is the usual shear parameter, $H_{n}$ is the

Hermite polynomial of order $n_{r}$ and $M_{n} \equiv(\pi / \sigma)^{1 / 2} 2^{n} n !$. The operator

$$
\frac{T_{e} e_{y} \hat{s}}{e n} \int_{-\infty}^{\infty} d x h_{n},(x)
$$

is then applied to Eq. (23) to qive the matrix equation 


$$
\sum_{n=0}^{\infty} I_{n n}(w) \bar{\phi}_{n}=0 \text {, }
$$

where

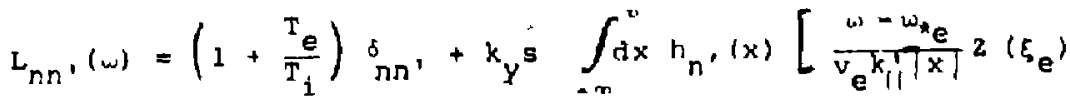

$$
\begin{aligned}
& +\frac{\omega_{*}^{n} e}{v_{e} k_{1}^{1}|x|}\left(\frac{1}{2} z\left(\xi_{e}\right)-\xi_{e}-r_{e}^{2} z\left(\xi_{e}\right)\right) \\
& +\frac{T_{e}}{T_{i}}\left(\frac{1}{v_{i} k_{l}^{T}|x|} \geq\left(\xi_{i}\right)(2 \pi)^{-1 / 2} \int_{-i n}^{n} d k_{x} \exp \left(i k_{x} x\right)\right. \\
& \times\left\{\left[w-w_{i}\left(1-\frac{3}{2} \eta_{j}\right)\right] r_{0}-w_{i}{ }_{i}\left[r_{0}+b\left(r_{1}-r_{0}\right)\right]\right\} \\
& -\frac{\omega_{\star}{ }^{\eta} i}{v_{i} k_{i}^{\prime}|x|}\left[x_{i}+\xi_{i}^{2} z\left(\xi_{i}\right)\right](2 \pi)^{-1 / 2} \int_{-x}^{\infty} d k_{x} \exp \left(i k_{x} x\right) \\
& \left.\left.x \Gamma_{0}\right)(2 \pi)^{-1 / 2} \int_{-\infty}^{\infty} d x \exp \left(-i k_{x} x\right)\right] \quad h_{n}(x) .
\end{aligned}
$$

In practice, the summation in Eq. (26) will be truncated to a finite number of terms, N. The complex constant o in Eq. (25) can be adjusted to minimize $N$, with the requirement that $\operatorname{Re}(\sigma)>0$, so that $\underset{x \rightarrow \pm \infty}{\lim } \tilde{\phi}(x)=0$. The basis functions given in

Ey. (5) have the useful property that their Fourier transforms 
are rnown analytically

$$
(2 \pi)^{-1 / 2} \int_{-\infty}^{n} \operatorname{dx} \exp \left(-i k_{x} x j h_{n}(x)=\frac{1-i)^{n}}{1 / 2 k_{y} s}-h_{n}\left(\frac{k^{k} x}{-k_{y}^{2} s^{2}}\right)\right.
$$

so that Eg. (27) can be rewritten as

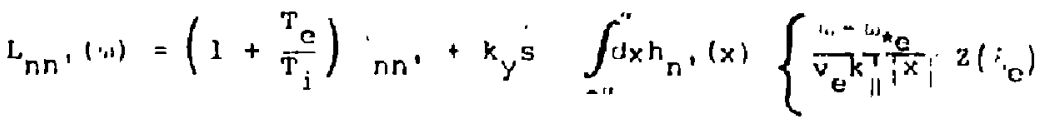

$$
\begin{aligned}
& \times h_{n}(x)+\frac{x_{c}{ }^{\prime} c}{v_{c} k_{i} \mid x}-\left(\frac{1}{2} 7\left(i_{e}\right)-i_{c}-r_{c}^{2} z\left(i_{0}\right)\right) h_{n}(x)
\end{aligned}
$$

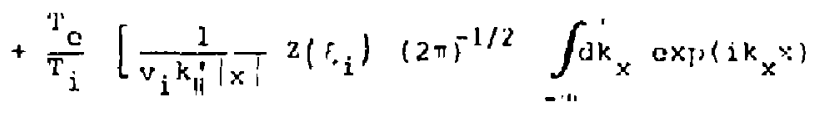

$$
\begin{aligned}
& \times\left\{\left[\omega-w_{i}\left(1-\frac{3}{2} r_{i}\right)\right] r_{0}-w_{*_{1}} \eta_{i}\left[r_{1}+b\left(r_{1}-r\right)\right]\right\}
\end{aligned}
$$

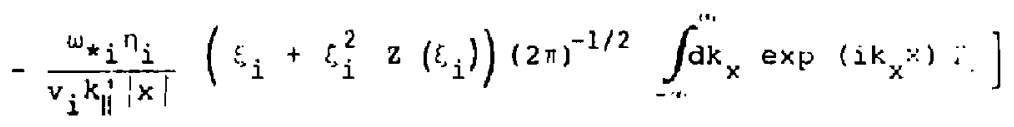

$$
\begin{aligned}
& \left.\times \frac{(-i)^{n}}{\sigma^{1 / 2} k_{y} \hat{s}} \quad h_{n}\left(\frac{k_{x}}{\sigma k_{y}^{2} \hat{s}^{2}}\right)\right\} \text {. }
\end{aligned}
$$

Hence on $l_{y}$ the $x$ and $k_{x}$ integrals need to be performed numerically when evaluating $\mathrm{L}_{\mathrm{nn}}$.. The numerical method used is high arder GaussHermite quadrature ${ }^{6}$. However, for the electron $x$ integration, $a$ separate Gaussian quadrature formula is used in the inner layer 
$x \cdot 2 x_{e} \equiv 2 ! w: /\left(k_{i}^{\prime} v_{e}\right)$. The numerical method used to determine the eigenvalue of $w$ for a selected eigenfunrtion is exactly that described in detail in Ref. 7 for the corresponding toroidal problem.

The usual second-order differential eynation may be obtained itom Er. (23) by expanding the Bessel functions in Eq. (21) through second order in $k_{x_{1}} \cdots 1$, leaviug $k_{y} \rho_{1}$ arbitrary. specifically

$$
:(b)=:\left(b_{y}\right)+\left[\Gamma_{1}\left(b_{y}\right)-\Gamma_{.}\left(b_{y}\right)\right) b_{x} \cdot
$$

and

$$
\begin{aligned}
& L\left[r_{I}(b)-\because_{0}(b)\right]=b_{y}\left[r_{1}\left(b_{y}\right)-r_{0}\left(b_{x}\right)\right]-r_{0}\left(b_{y}\right) \\
& \left.+2 b_{y}\left(r_{1}\left(b_{y}\right)-r_{0}\left(b_{y}\right)\right)\right] b_{x} .
\end{aligned}
$$

With appropriate integrations by parts, the integrals in Eq. (21) may then be performed to yield the familiar second order differential equation ${ }^{1-4}$ governing long radial wavelength drift eigenmodes. 


\section{ELECTRON DRIFT EIGENMODES}

As noted in the preceding section, the spatzal yartat.cons in the equijibrium quantities is taken tro be much weaker than the variations on the scalc of the perturbations. Within the rrame work of this familin ordering it has been demonstraled that collisionless electron drift waves ("universal modes") arr absolutely stable at long radial wave lengths iu a shcared slab geometry. However, for larger azinuthal wavenumbers $\left(k_{y^{i}}{ }^{*} 1\right)$, the corresponding tighter radial localitation of the eigenmorles (discussed in sec. I) forces a consideration of shorter radial wavelengths. Since the differential equation formulation break. down as $k_{x} n_{i}+1$, it: becomes necessary to solve the inteyral eigenmode equation to determine the behavior of thesc waves in the shorter wavelength part of the spoctrum.

As described in Sec. II-B, we have devcloped a computer code which implements the tasis function, or Ritl, method to solve the eigeamode equation of interest. Ear the analysis of universal modes, this numerical procedure is limited by the requirement that there are two distincily different langth scales in the problem. Specifically, it is necessary to choose a sufficient number of appropriate basis functions to cover both the small-scale region around the average electron resonance point, $x_{e} \equiv|w| / k_{1}^{\prime} y_{e}$, as well as the large-scale region out to the average ion resonance point, $x_{i} \equiv|\omega| / k_{\|}^{\prime} v_{i}$. Tn properly determine the stability properties of these modes it has been emphasized in the differential equation studies ${ }^{1}$ that it is 
ussential to account for the detailed behavior around the narrow cjcctron fesonance region. Since the ratio, $x_{i} / x_{e}=\left(m_{i} T_{e} / m_{e} T_{i}\right)^{1 / 2}$, is obviously very large, the required number of basis functions, $N$, becolies correspor. Hirqly prohibitive for realistic mass ratios. In farticular, $i \cdot$ is found that the largest ratio, $x_{i} / x_{e}$ that can be practically handled is roughly equal to $N^{1 / 2}$. Hence, in the cases studied by this numerical procedure, we are restricced to artificially snall mass ratios.

is a check on the integral formulation code, we considered sume moderate wavelength cases $\left(k_{y} p_{j}: 1\right)$ which are nea: the limits of validity of the differential formulation. For a reprosentative use with $m_{i} / m_{e}=100, k_{y} \rho_{i}=0.63, L_{s} / L_{n}=100$, $\mathrm{T}_{\mathrm{c}}=\mathrm{T}_{\mathrm{i}}$, $\mathrm{l}_{\mathrm{e}}=\mathrm{l}_{\mathrm{i}}=0$, and $0=1.33$, we obteined an eigenvalue of w/u*e $=0.2261+19.6 \times 10^{-3}$ using 120 basis functions. Although not as accurate, this result compares reasonubly wol] with a differential shooting code calculation of the same case using 500 equally-spaced grid points in $x .{ }^{9}$ specifically, the shootin, code result is $\omega / \omega_{k}=0.2294+i 5.0 \times 10^{-5}$. The even eigenfunction and its Fourier transform, $\dot{\phi}\left(k_{x}\right)$, calculatr $d$ by the integral formulation code for this case are shown on Fig. 1 . Here it is seen that, as expected, the eigenfunction is evanescent beyond the ion Landau resonance region, $|x|>x_{i}$. It is also of interest to note that the Fourier transformed eigunfunction for this moderately short wavelength case is dominated by values of $k_{x} \rho_{i}$ around 2 . 
To illustrate the qualitative behavior of the undr:sal mode of shortnr wavelengths, we now considre a representatire case with ${ }^{11_{1}} / m_{c}=100, K_{v} r_{i}=0.71,1_{s} / L_{n}-56, \gamma_{r}-r_{1}$, $n_{i}=r_{c}=0$, and $r=0.75$. On Fir. 2 , the nirgonvalue w (normalized to "a) is shown as a function of 11 fer ene ceven cigenfungtion. The results herredrmonstrate that a; the proper number of basid: functions required to describn the aifenfunetuon is approarbed, the corresponding cigenvalue bromes that of a damped normai mote. In this, as well as for id: of the other cases examined, we have found no eridence for the rivgelspment of unstable universal cigennodes at the shorter rubiai wuvelengths where differences between the integral uni diiforutial formulations aro significant. It should be notcel, howrver, that those results il: themsejves do not constitute a mathemalical proof for the non-existence of unstable short wavelenglh iniversul modes.

The lack of numerical efficiency of tho latz method urocodure for dealing with the universal modes is not difficult to understand. Since the basis functions die of $\mathrm{f}$ rapily in : boyond the width of the highest-n basis function kept, their truncation effectively forces the eigenfunction to zero at a very short distance beyond this $x$-value. The requirement then is that this point must fall well outside the ion Lanrau damping region $\left(x \simeq x_{i}\right)$, where the wave energy is absorbed. If this criterion is not met, the artificial forcing of the eigenfunction to zero 
introduces corresponding spurious refiection of the outgoing chergy. As a result, incorrect positive growth rates of the type shown in Fig. 2 can be reatily cenerated.

Motjvated by the preceding considerations, wo have also analyzed the integral equation problem by a wkH jroccdurs. As driscribec in etail in Ref, 10, this involved the introduction of an ordering in poweis of $c-p_{i} / L_{n}$ far crom the relevant Lurning points and in powers of $\mathrm{c}^{1 / 3}$ close to such turning points. Matching the solutions in the different regions then leads to Lho familiar form of the pisase-inteqral eigenvalue coudition:

$$
\int_{x}^{x_{x}} d x x_{x}(x, \omega)=(p+1 / 2) \tau
$$

where $r=0,1,2,$. . and $k_{x}(x, w)$ is determined by the solutius of the lowest order dispersion relation:

$$
\Gamma_{0}\left[\left\{k_{x}^{2}+k_{y}^{2}\right) \frac{0_{i}^{2}}{2}\right]=-\frac{1+\frac{T_{e}}{T_{i}}+\left(1-\frac{\omega_{k}}{\omega}\right)\left(\xi_{c}\right) z\left(\xi_{c}\right)}{\left(\frac{T_{e}}{T_{i}}+\frac{\omega_{\star} e}{\omega}\right)\left(\xi_{i}\right) z_{(}\left(\xi_{i}\right)} .
$$

Temperature gradients are ignored here for simplizity, and the turning points, $\ddot{i}_{1}$ ard $x_{2}$, are determined by

$$
k_{x}\left(x_{1}, w\right)=k_{x}\left(x_{2}, w\right\}=0 .
$$

This generally leads to complex values for $x_{1}$ and $x_{2}$. 
The phase-integral procedure outlined here has bocn numerically implemented ${ }^{11}$ using an interactive code!? Whon the appropriate evanescent boundary conditions are imposed, there is again no evidence found for the existence of unstable eigenmodes at short wavelengths. However, as noted earlinr, thesr numerical studies do not prove in a mathematically definit.ve sense that such modes cannot exist.

IV

ION DRIFT EIGENMODES

Unlike the situation for universal modes, the aupliration of the Ritz nethod to the integral equation problen. lor ion drift waves is quite efficient. The obvious reason is that the small-scalc effects associated with the electron resonance region $\left(x=x_{e}\right)$ are unimportant for these eigenmodes. This fact is clearly demonstrated by the differential equation. Ludies of long wavelength ion tempcrature-gradient-driven urift instabilities. Results from a shooting code analysis of a typizal case (with parameters $L_{s} / L_{n}=20, T_{e} / T_{i}=2, \eta_{i}=\eta_{e}=2$, and $k_{y} o_{i}=1$ ) ?-e displayed in Fig. 3. Here the eigenvalue, $w$, is calculated as a function of the mass ratio. For unrealistically large ratios, such as $m_{e} / m_{i}=1 / 100$, the electron resonances can introduce substantial shifts in the eigenvalue. However, for realistic values, such as $m_{e} / m_{i}=1 / 3672$ or $1 / 1836$, the eigenvalue is negligibly shifted from that obtained assuming purely adiabatic electrons, i,e. $m_{e} / m_{i}=0$. Hence, to a very good 
approximation, the non-adiabatic effects associated with electron resonances can be ignored for these modes.

Using the adiabatic response for electrons, $\bar{n}_{e}=\left(e \bar{\phi} / T_{e}\right)$, and again considering the case described in preceding comments. we calculatew the eigenvalue as a function of the aximuthal wave number $k_{y^{\prime}}$ or $b_{y} \equiv k_{y}^{2} p_{i}^{2} / 2$, with a shooting colle. $\lambda$ s ilustrated or. Ficj. 4, the results are in agreament with carlior calculations ${ }^{2}$ showing that the growth rate has a maximum near $b_{y}=0 . B$ and that the mode fieqlency continues to increase beyond $b_{y}=1.0$. However, at sucli relatively short wavelengths the differential equation used to lerive thrse results is no longer valid. In what follows we will consider only the proper integral equation analysis of these modes.

Since the present eigenmode problem has only one spatial scale of interest, the kitz method proves to be very efficient for obtaining solutions to the governing integral equation. Taking the parameters of the previous casc solved with the shooting code, we used $\sigma=1.0$ and $N=120$ in the integral formulation code to obtain the eigervalue, $\omega / \omega_{*_{e}}=\omega \tau_{s}=$ $-0.036+i 0.029$, for $b_{y}=0.5$ (i.e., $k_{y} p_{i}=1.0$ ). The differential equation result, $\omega / \omega_{\star_{e}}=-0.043+i 0.043$, differs by about $20 \%$ in the real frequency and 508 in the growth rate. Agreement between results from the two codes is, of course, better at longer wavelengths. In the present case, however, it can be concluded that the non-regligible shifts in the eigenvalue are due to inaccuracies introduced by the 
differential approximation. This point is best illustrated by Fig. 5, where the corresponding even eigenfunction and it: Fourier transform, $\phi\left(k_{x}\right)$, are plotted for this case. Although the root-mean-square value of $k_{x} p_{i}$ is of the same order as $k^{\prime \prime}{ }_{i}$ 1, it is clnar Crorl lij. S that the actual distribution in $k_{x}$ for this morle cytnnds to scveral times $k_{y}$ with arpreniable ampliturle. The dopendence of thr eigenvalue, $w$, on the azimuthal wavenumber $\left(b_{y}\right)$ and the ion-temperature gradient $\left(\eta_{i} ; l_{1}\right.$ as also been investigatod with the integral formulation code. Ot fig. 6 it is seen that the maximum growth rate occurs for $b_{y}$ between 0.4 and 0.5 , and that the real Erequency, $-\omega_{r} \tau_{s}$, reaches a plateau at by:0.7. This differs significantly from the differential formulation results displayed in Fig. 4 which indicated maximum growth at $b_{y}=0.8$ and a continuing increasing trend for the real frequency beyond $b_{Y}=1.0$. On the other hand, the integral equation results for the $\eta_{i}$ dependence of the eigenvalue displayed in Fig. 7 is in general qualitative agreement with the differential equation analysis. Botls types of calculations indicate an instability threshola at $\eta_{i} ? i$.

v. CONCLUSION

In this paper we have presented a systematic derivation of the appropriate integral eigenmode equation for analyzing drift waves of arbitrary radial wavelengths in a sheared slab system. For collisionless electron drift waves ("universal" modes) our main cunclusion is that although we cannot definitely 
ride out the possible existence of short wavelength absolute instabilities of this type, no evidence has been found to support their existence. As a practical point we should also note that the Ritz method is inefficient for dealing with the universal mode problem because of the stringent requirement that the basis functions used must properly cover the two widely different spatial scales associated with the electron and ion resonanc. . gions. However, for the ion-temperature-gradientdriven ion drift instabilities, the Ritz procedure is quite afficient and allows us to readily explore the behavior of these modes throughout the spectrum. This is due to the fact that the electron resonance effects can be ignored for these irstabilities. Our main conclusions for the "i modes are: (1) even at short wavelengths $\left(k_{y} p_{i} \geq 1\right)$, the instability threshold remains at $\eta_{i} \geq 1 ;(2)$ the maximum growth rate for these modes occurs around $k_{y} \rho_{i} \simeq 1$; and (3) the real frequency reaches a plateau near $k_{y} \rho_{i} \geq 1$ instead of following the monotonically increasing trens exhibited by the differential equation results.

\section{ACKNOWLEDGMENTS}

We would like to thank R. B. White for the use of his interactive WKB-shooting code.

This work was supported by the United States DoE Contract No. $E Y-76-C-02-3073$. 
REFERENCES

1.D. W. Ross and S. M. Mahajan, Phys, Rev. Lett. 40, 324 (1978); K. T. Tsang, F. J. Catto, J. C. Whitson, and J, Smith, Phys. Rev. Lett. $\underline{40}, 327$ (1978); L, Chen, P.N. Guzdar, R, B. White, P.K. Kaw, and C. Oberman, Phys. Rev. Lett. 41. 549 (1978).

2. R. Waltz, W. Pfeiffer, and R. R. Dominquez, General itomic Report $G \Lambda-\lambda 15147$ (1978).

3. W. M. Tang, R. B. White, and P. N. Guzajar, Princeton Univorsity Plasma Physics Laboratory Report PPLL-1541 (1979).

4.W. M. Tang, Nucl. Fusion 18, 1089 (1978).

5.E. C. Titchmarsh, Introduction to the Theory of Fourier. Integrals (Clarendon, London, 1948), p. 81 .

6.A. H. Stroud and D. Secrest, Gaussion Quadrature Formulas (Prentice Hall, Englewood Cliffs, 1966).

7.G. Rewoldt, W. M. Tang, and E. A. Frieman, Phys. Fluids 21, $1513(1978)$.

8.T. Antonsen, Phys. Rev. Lett. 41, 33 (1978). 
9. Ross, Private Communication (1978).

10.E. A. Frieman, G. Rewoldt, W. M. Tang, and A. H. Lilasser, Princeton University Plasma Physics Laboratory Report PFPL$1560(1979)$.

$11+w$, w. Lee, Private Communication (1978).

12.R. B. White, J. Comput. Phys. 31, 409 (1979). 

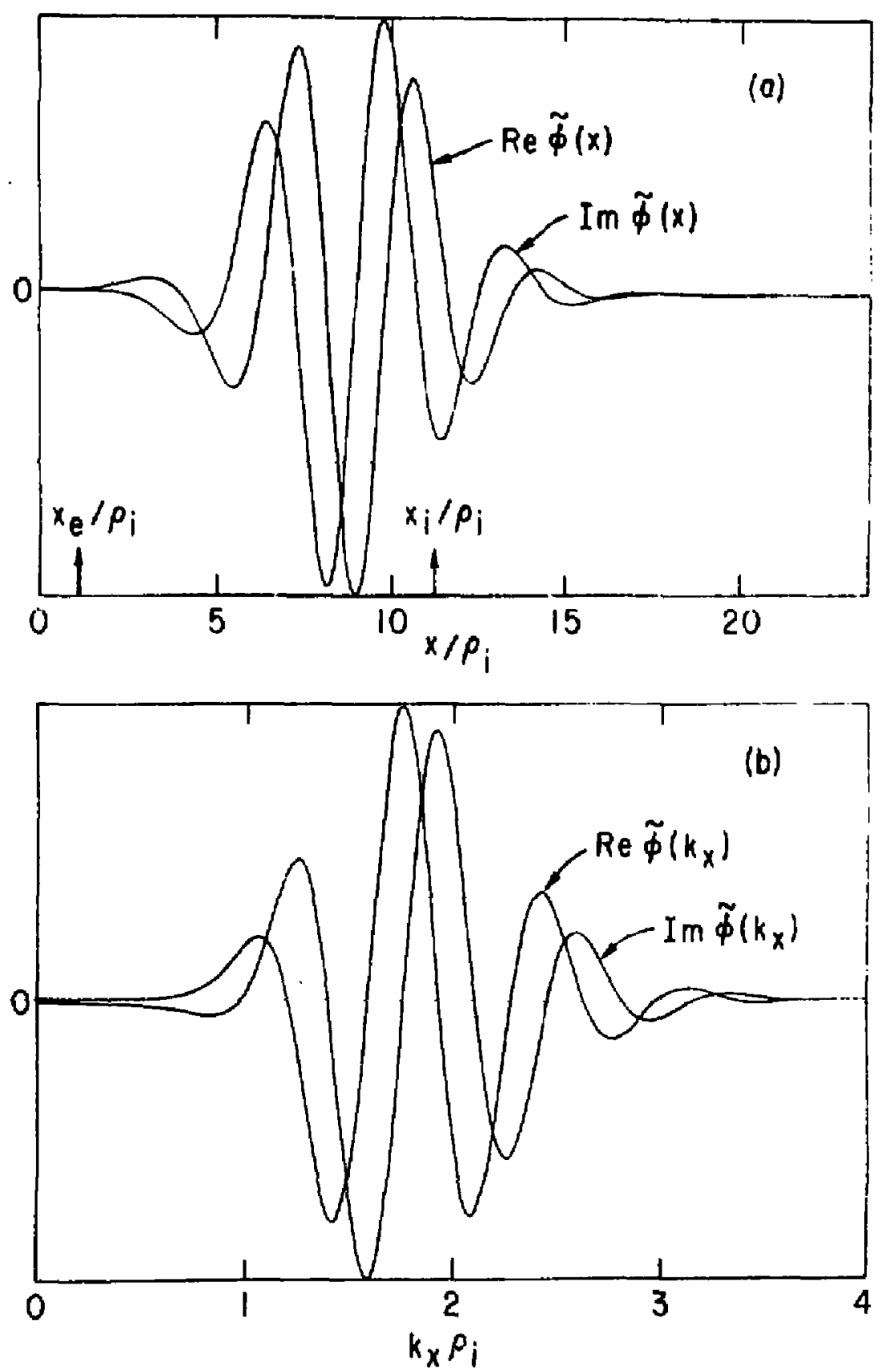

792324

Fig. 1. Eigenfunction $\bar{\phi}(x)$ and Fourier transform $\bar{\phi}\left(k_{x}\right)$ from the integral formulation code for the parameters: $k_{y} \rho_{j}=0.63, L_{s} / L_{n}=100, m_{i} / m_{e}=100, T_{e} / T_{i}=1$, $n_{i}=n_{e}=0, \sigma=1.33+0 i$, and $N=120$. The eigenvalue is w/we $=0.2261+0.0096 i$. 


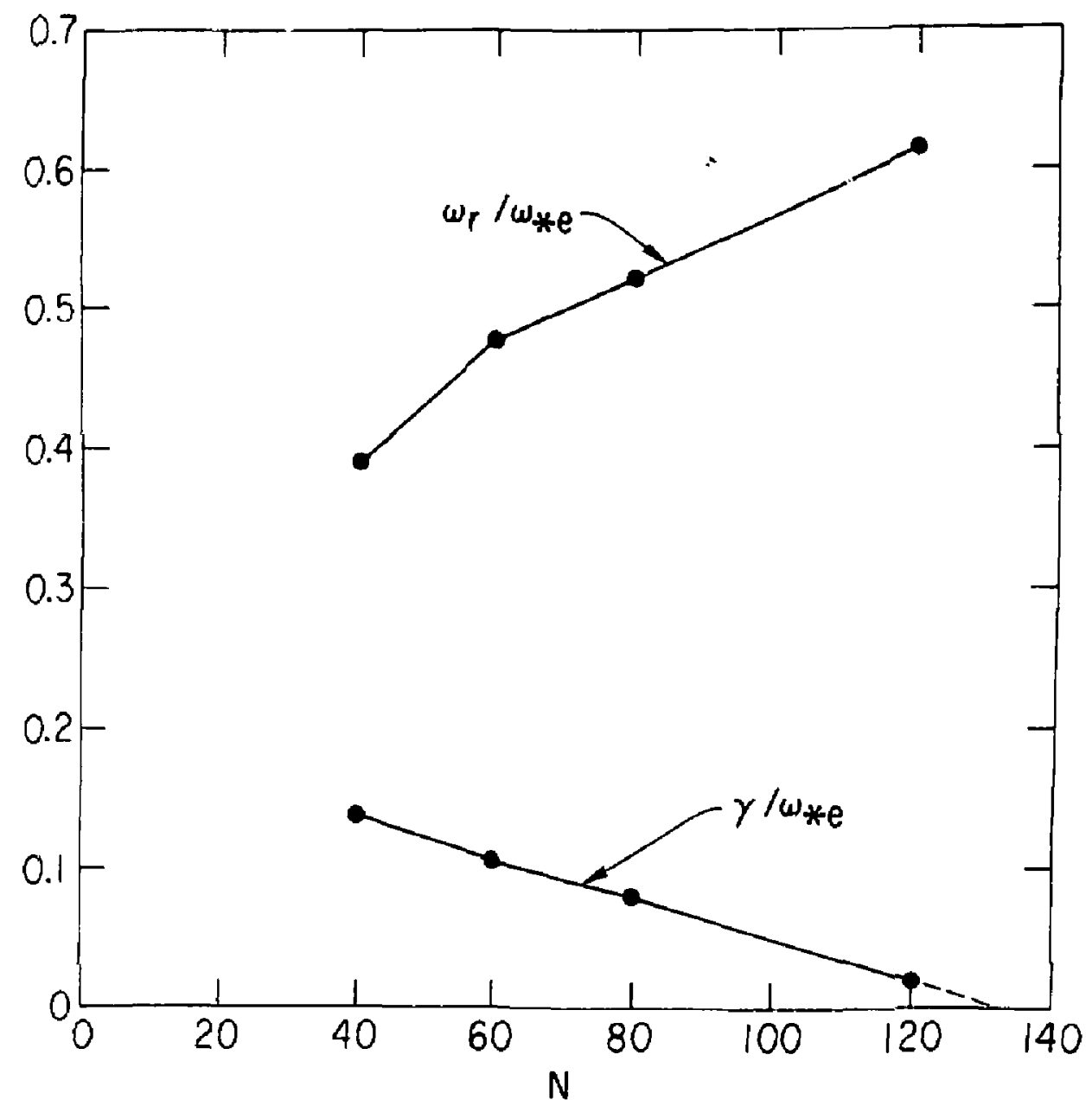

792312

fig. 2. Real and imaginary parts of the eigenvalue $\omega \equiv \omega+$ iy versus $N$, the number of basis functions used in the integral formulation code. Th, parameters are: $k_{y} D_{i}=0.71, L_{s} / L_{n}=56, n_{i} / m_{e}=100, r_{e} / T_{i}=1, n_{i}=n_{e}=0$, and $a=0.76$ $+0 i$. 


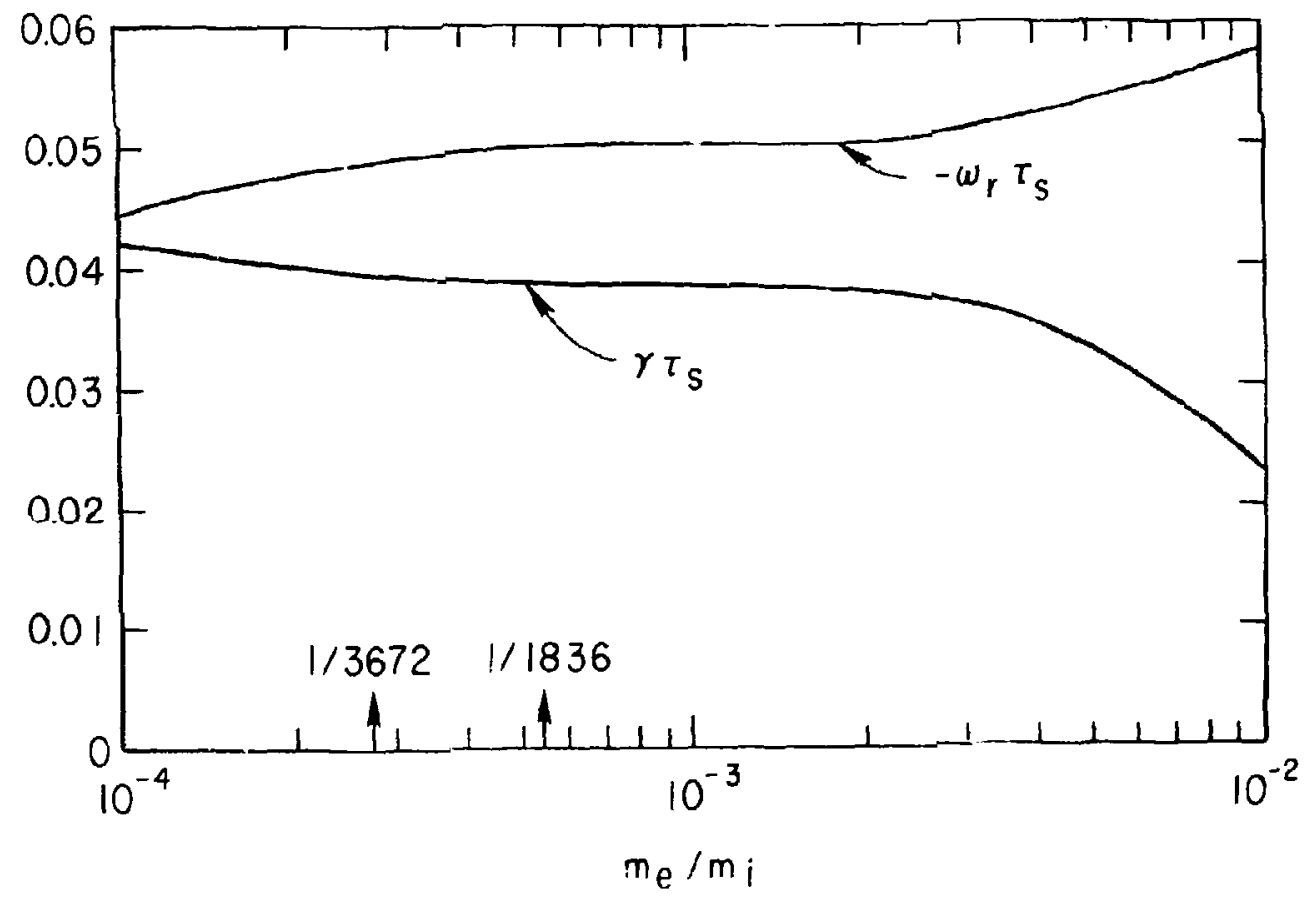

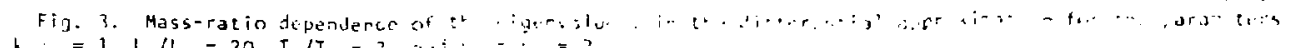

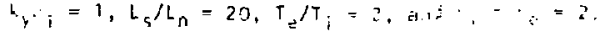




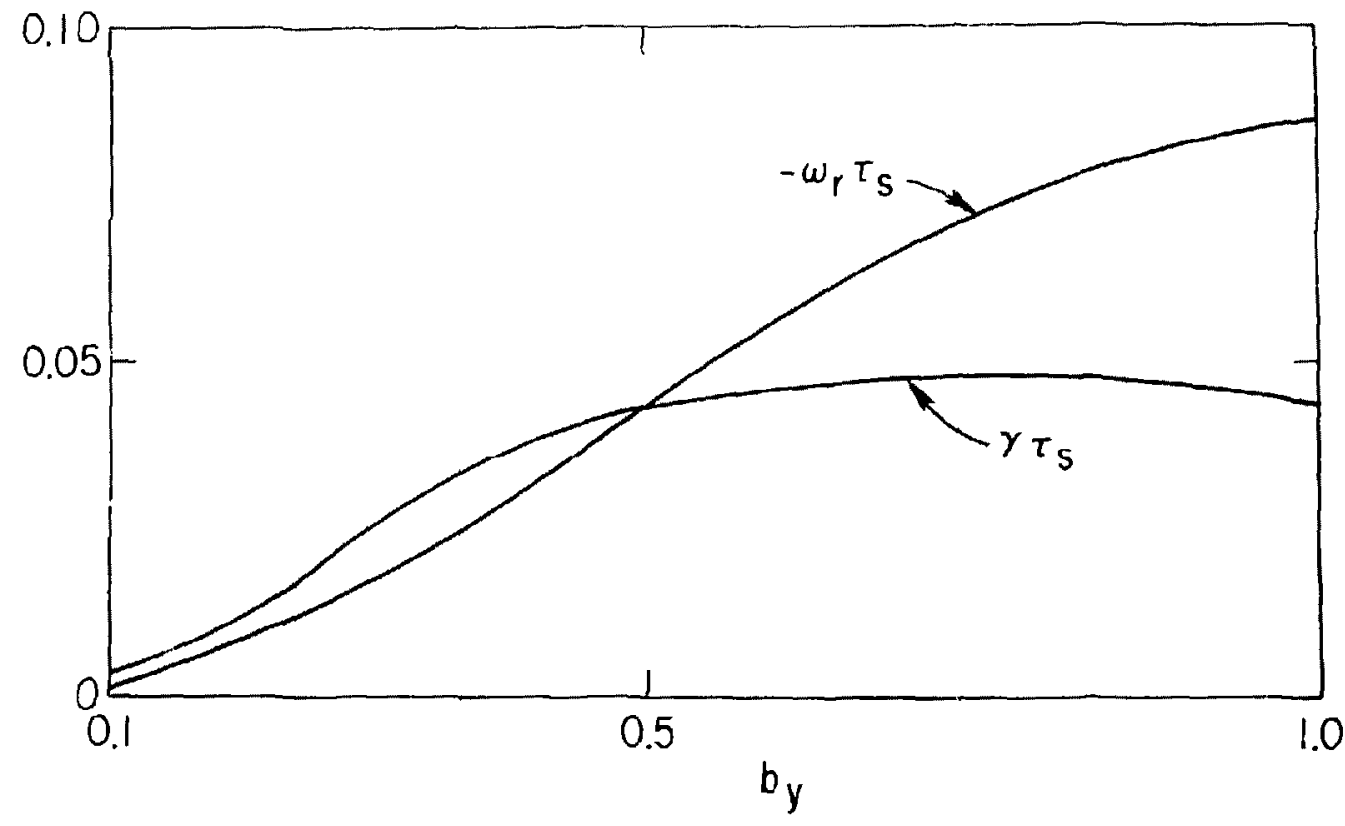

Fig. 4. Dependence of the eigenvalue on $b=k^{2} 2 / 2$. 192459 electron response for the parameters $L_{s} / L_{n}=20,7 Y / T_{i}=2$, and $\tau_{i}=2$. 


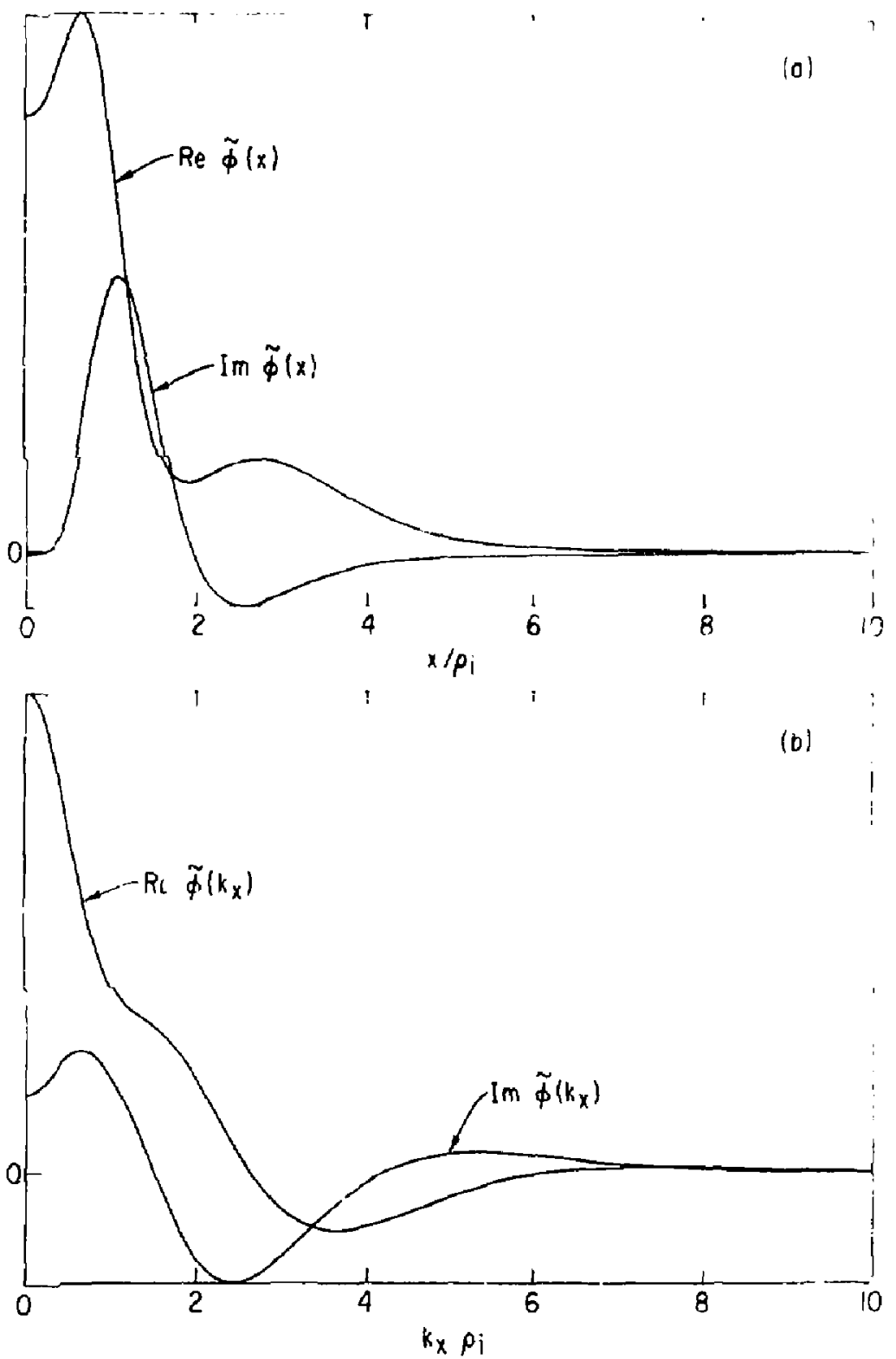

Fig. 5. Eigenfunction $\tilde{\phi}(x)$ and Fourier transform $\dot{\phi}\left(k_{x}\right)$ in the integral formula$t$ ion with adiabatic electron response for parametirs: $b_{y}=0.5, L_{s} / L=20, T / T$, $=2, \eta_{i}=2, \sigma=1+0 i$, and $N=120$. The e igenvalue is ${ }^{y} / \omega_{\text {ite }}=-0.836+0.029 i$. 


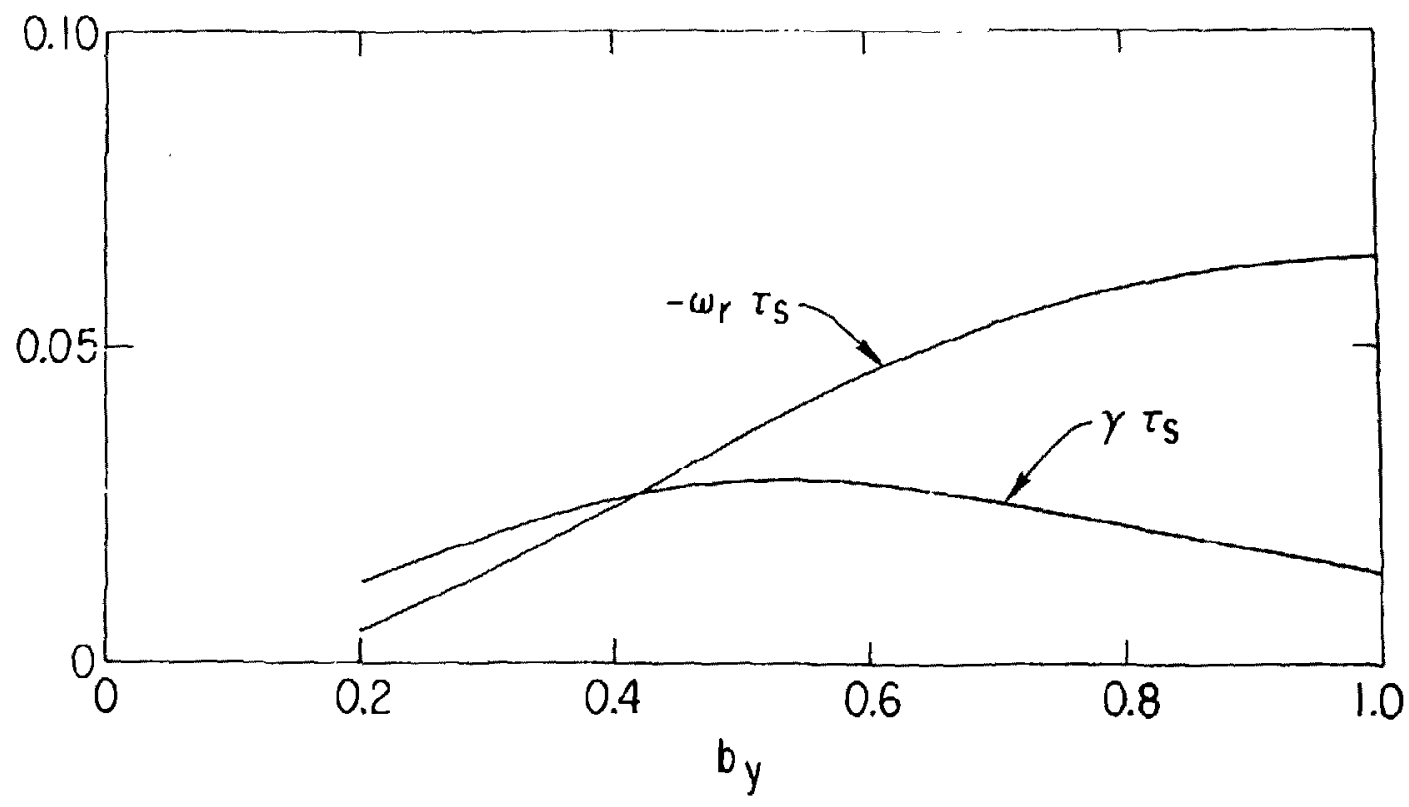

Fig. 6. Feal and imaginary parts of the eigenvalue. 792314 adiabatic electron response, with the parameters:

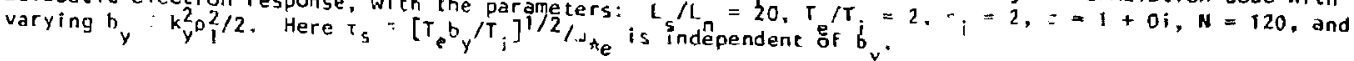




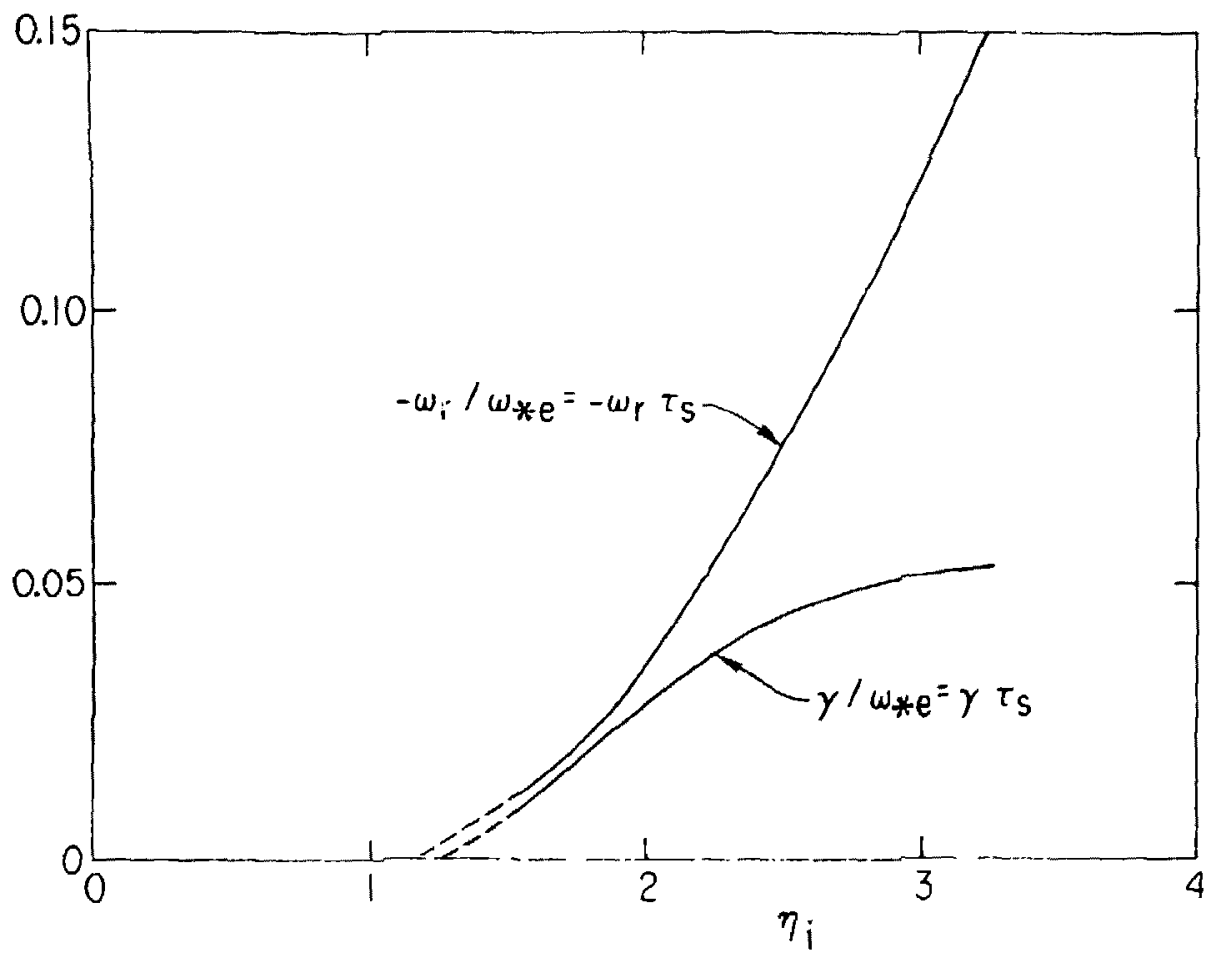

i

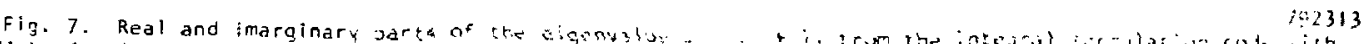

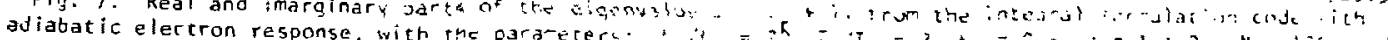
varying $?_{i}$. 